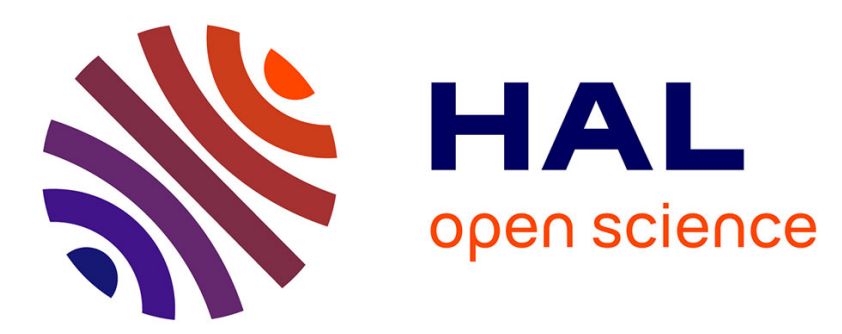

\title{
LMPC based online generation of more efficient walking motions
}

\author{
Andrei Herdt, Nicolas Perrin, Pierre-Brice Wieber
}

\section{To cite this version:}

Andrei Herdt, Nicolas Perrin, Pierre-Brice Wieber. LMPC based online generation of more efficient walking motions. IEEE-RAS International Conference on Humanoid Robots (Humanoids), Nov 2012, Osaka, Japan. pp.390-395, 10.1109/HUMANOIDS.2012.6651549 . hal-02487630

\section{HAL Id: hal-02487630 \\ https://hal.inria.fr/hal-02487630}

Submitted on 21 Feb 2020

HAL is a multi-disciplinary open access archive for the deposit and dissemination of scientific research documents, whether they are published or not. The documents may come from teaching and research institutions in France or abroad, or from public or private research centers.
L'archive ouverte pluridisciplinaire $\mathbf{H A L}$, est destinée au dépôt et à la diffusion de documents scientifiques de niveau recherche, publiés ou non, émanant des établissements d'enseignement et de recherche français ou étrangers, des laboratoires publics ou privés. 


\title{
LMPC based online generation of more efficient walking motions
}

\author{
Andrei Herdt ${ }^{1}$ and Nicolas Perrin ${ }^{2}$ and Pierre-Brice Wieber ${ }^{3}$
}

\begin{abstract}
This paper proposes solutions to the relative lack of efficiency of walking trajectories generated online. Extensions of a previously introduced Linear Model Predictive Control law are proposed that allow to improve the walking performance through variation of the height of the CoM and toe flexion. Extensive simulations serve to demonstrate the capacity of the improved scheme to generate significantly more efficient walking motions.
\end{abstract}

\section{INTRODUCTION}

Various online walking motion generation schemes have been developed during the last decade that allow to control the displacement of biped robots [1][2][3][4][5][6]. Relying on linear dynamics, these schemes generate walking motions that lack by far the efficiency and naturalness of human motions. Like all of the approaches mentioned above, the Linear Model Predictive Control (LMPC) scheme that we introduced in [7] makes the assumptions that the motion of the Center of Mass (CoM) is limited to a horizontal plane and that the support foot does not rotate around the heel or the toe during the entire support phase, i.e. foothold is always held with the entire sole. To circumvent the resulting restrictions on the motion of the robot, the height of the CoM has to be reduced, leading to the walk with strongly bent knees that is characteristic to most humanoid robots today.

Biomechanical studies highlight a walking pattern that differs in several points from the one generated by these schemes:

(a) The support leg is kept straightened (although not completely straight) during the entire stance phase.

(b) Toe flexion extends the effective leg length at the end of single support phases.

(c) The height of the CoM follows a sinusoid-like curve with the lowest position in the middle of the double support phase and the highest in the middle of the single support phase.

We know that this pattern is energetically advantageous [8][9][10][11][12][13][14][15]. Little muscle force is needed to support the body if the knee is kept straightened, but overstating point (a) leads to stiff legs and invertedpendulum-like walking motions that increase the vertical velocity at the end of single support phases [16] and consequently the impact forces together with the amount of positive and negative mechanical work that is necessary

\footnotetext{
${ }^{1}$ A. Herdt is with the Institute of Robotics and Mechatronics, DLR German Aerospace Center, Wessling, Germany. andrei.herdt at dlr.de

${ }^{2}$ N. Perrin is with the Advanced Robotics, Istituto Italiano di Tecnologia, via Morego, 30, 16163 Genova. nicolas.perrin at it. it

${ }^{3}$ P.-B. Wieber is with the INRIA Grenoble - Rhone-Alpes, France. pierre-brice.wieber at inria.fr
}

to redirect the Center of Mass during the double support phase. Both the production of positive and negative work requires energy expenditure in human muscles. The extension (b) of the stance leg during the redirection phase reduces this vertical velocity of the CoM together with the work that is necessary to redirect it to a new single support phase [17][18]. These two characteristics, almost straight-leg and toe flexion, are the major reasons for the sinusoid-like evolution (c) of the CoM.

Even if evidence exists that the above two strategies increase the efficiency and performance of human walking motions, the same conclusion cannot be directly applied to robots. Joint actuators might be capable of producing electric power from negative mechanical work and energy can be stored for longer periods than in the human body. However, straight leg walking obviously alleviates the load on the joints by reducing the velocities and torques, especially in the knees. And toe flexion allows increasing the achievable stride length, as well as the height of the CoM during the double support phase. Imitating the above features of the human walk is therefore likely to improve the performance of walking robots.

In this paper, we achieve the imitation of the above characteristics by our online trajectory generation scheme. In Section II, we show how variations of the CoM height can be achieved without losing the linearity of the horizontal motion equations. The proposed solution can be found in similar forms in [19][20][21], although either in a more restricting or a numerically less advantageous form. We address in Section III the geometric feasibility constraints that become important when the robot is walking with straightened legs. The offered solution is extended in Section IV to a criterion for a more efficient and versatile use of toes than what can be found in the literature, for example in [19][22][23][24][25].

\section{VERTICAL MOTION OF THE COM}

The motion of the CoM $c$ of a robot walking on a perfectly horizontal ground is linked to the position of the Center of Pressure $(\mathrm{CoP}) z$ on the ground by the following nonlinear second order differential equation:

$$
c^{x, y}-\frac{c^{z}}{\ddot{c}^{z}+g} \ddot{c}^{x, y}=z^{x, y}
$$

with $g$ the gravitational acceleration constant of $9.81 \mathrm{~m} / \mathrm{s}^{2}$.

This equation is linear with respect to the horizontal motion $c^{x, y}$ and even Linear Time-Invariant (LTI) when the height $c^{z}$ of the CoM is fixed. In this case, we can define a Linear Receding Horizon Scheme (LRHS) where minimizing the deviation of the horizontal CoM velocity 
$\dot{c}^{x, y}$ with respect to a reference $\dot{c}_{r e f}^{x, y}$ over a short period of future time is enough to obtain stable and reactive walking motions [7]:

$$
\operatorname{minimize} \sum_{i=1}^{N}\left(\dot{c}_{i}^{x, y}-\dot{c}_{i, r e f}^{x, y}\right)^{2},
$$

with $N$ the number of samples $i$ with a fixed period of 100 ms considered over the duration of two steps.

Feasibility requirements on the contact forces can be considered by a set of inequalities on the position of the CoP:

$$
E_{z}\left(p_{i}^{\theta}\right)\left(z_{i}^{x, y}-p_{i}^{x, y}\right) \leq e_{z}
$$

which is linear with respect to $z$ and the position $p^{x, y}$ of the support foot on the ground, but where the matrix $E_{z}\left(p^{\theta}\right)$ varies nonlinearly with respect to the orientation $p^{\theta}$ of the support foot.

The feasibility of leg motions can be ensured in a similar way by inequalities on subsequent foot placements $p_{j}, p_{j+1}$ :

$$
E_{p}\left(p_{j}^{\theta}\right)\left(p_{j+1}^{x, y}-p_{j}^{x, y}\right) \leq e_{p},
$$

that prohibit crossing the feet (and the subsequent risk of self-collisions) as well as over-stretching the legs. More details about these formulas can be found in [7].

In what follows, we exploit the fact that when the height of the CoM varies as a predefined function of time $t \rightarrow c^{z}$ equation (1) becomes Linear Time-Variant (LTV), what can be handled equally well with the same control scheme (cf. Section VI).

But obviously, predefining the vertical motion of the CoM must be done with care: Frequency and amplitude have to be synchronized with the step duration and length. With the periods of the different support phases fixed in advance, as already required in [7], the synchronization with the step sequence can be achieved easily. To obtain a desired gain in efficiency, however, the amplitude of the vertical motion has to be chosen depending on the stride length and the walking direction, making the use of heuristic adaptation strategies necessary. A database of predefined trajectories for such a heuristic adaptation scheme can be obtained with a comprehensive set of simulations, what we will do here in Section V.

\section{ENSURING GEOMETRIC FEASIBILITY}

Geometric feasibility is an important requirement for walking motions. The constraints (4) allowed to avoid selfcollisions and over-stretching of the legs but under very restrictive assumptions. When working with vertical motion of the CoM, they need a complete overhaul.

Given the position and orientation $p$ of the support foot, we can define the set of reachable CoM positions in the Cartesian space:

$$
c \in \mathcal{C}(p)
$$

In the following, we make the common assumption that when walking, the CoM of the robot is immobile with respect to the hip. A simple forward kinematics procedure taking into account only the kinematics of the support legs is then sufficient to compute this reachable set.

Figure 1 shows a sagittal cut through the set $\mathcal{C}(0)$ of the HRP-2 robot that has been computed in this way. Being nonlinear and nonconvex, this set cannot be considered directly inside our linear control scheme. The entire set, however, is not of equal interest for walking: Lower CoM positions lead to strongly flexed legs and consequently to high torques and high velocities in the knee joints, and the corresponding walk looks unnatural. We are interested therefore in CoM positions that lead to relatively stretched legs, i.e. the higher, convex part of the feasibility region (cf. Figure 1). Approximated by a convex polyhedron, this subset can be expressed by a set of inequalities linear with respect to the positions $c^{x, y, z}$ and $p^{x, y, z}$ of the CoM and support foot:

$$
E_{c}\left(p_{i}^{\theta}\right)\left(c_{i}^{x, y, z}-p_{i}^{x, y, z}\right) \leq e_{c}
$$

and nonlinear with respect to the support foot orientation $p^{\theta}$. For the HRP-2 robot that we used to obtain the results in Section V, an accurate approximation of the feasible area could be achieved by a polyhedron with only ten faces.

Since the vertical motion of the CoM is predefined, this geometric constraint needs only be checked with respect to the horizontal motion. Now, looking at Figure 1, we can see that satisfying the inclusion (5) only at the beginning and end $t_{D S}^{-}$and $t_{D S}^{+}$of the support phase is sufficient to ensure that it is satisfied for the whole motion inbetween.

Finally, we obtain the following optimization problem:

$$
\begin{aligned}
\text { minimize } & \sum_{i=1}^{N}\left(\dot{c}_{i}^{x, y}-\dot{c}_{i, r e f}^{x, y}\right)^{2} \\
\text { subject to } & \\
t_{i} \in\left[t_{1}, t_{N}\right] & c_{i}^{x, y}-\frac{c_{i}^{z}}{\ddot{c}_{i}^{z}-g} \ddot{c}_{i}^{x, y}=z_{i}^{x, y} \\
t_{i} \in\left[t_{1}, t_{N}\right] & E_{z}\left(p_{i}^{\theta}\right)\left(z_{i}-p_{i}^{x, y}\right) \leq e_{z} \\
& E_{p}\left(p_{j}^{\theta}\right)\left(p_{j+1}^{x, y}-p_{j}^{x, y}\right) \leq e_{p} \\
t_{i} \in t_{D S}^{+} & E_{c}\left(p_{i-1}^{\theta}\right)\left(c_{i}^{x, y, z}-p_{i-1}^{x, y, z}\right) \leq e_{c} \\
t_{i} \in t_{D S}^{-} & E_{c}\left(p_{i+1}^{\theta}\right)\left(c_{i}^{x, y, z}-p_{i+1}^{x, y, z}\right) \leq e_{c} .
\end{aligned}
$$

Note that the constraints (11) and (12) prohibit now the overstretching of the legs, but constraints of the form (10) are still necessary to prevent self-collisions. The constraints (11) and (12) can be rotated in order to consider rotations of the feet during the double support phase observed in human walking, but for reasons of simplicity, we keep the more restrictive form (6).

\section{PURPOSEFUL TOE FLEXION}

As mentioned in the Introduction, straight legs alone do not lead to more efficient or natural looking motions. Although straightening the stance legs lowers the load on the joints and allows for a greater horizontal velocity, it also increases the vertical velocity of the height of the CoM at the beginning and end of its redirection phase around the 


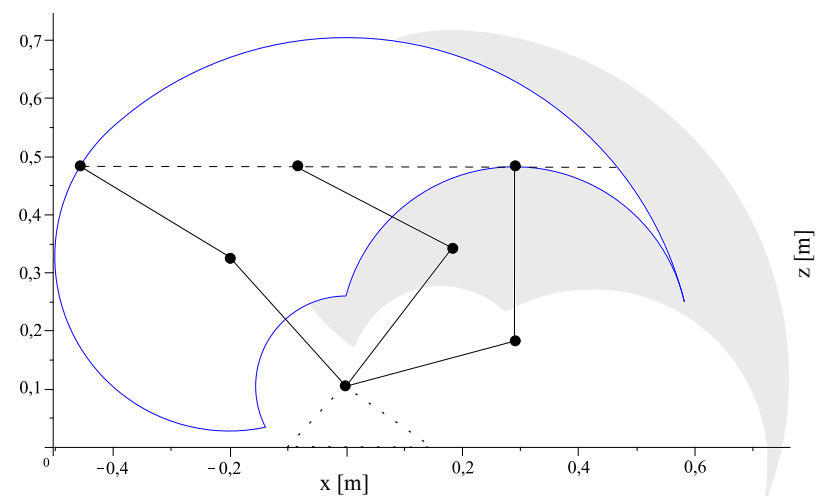

Fig. 1. Feasible CoM positions in the sagittal plane before and after (shaded region) a $45^{\circ}$ rotation around the toe. The dashed line maximizes the horizontal reach when walking with a constant CoM height. The area above leads to more 'comfortable' leg configurations that are, for this reason, more relevant for walking.

double support phase and therefore increases the required positive and negative mechanical work. This drawback can be circumvented by toe flexion at the end of the single support phase, what allows to increase the effective length of the stance leg at this period and thus reduce the vertical oscillation of the CoM. The question then is how to assure that the generated walking motions exhibit this feature, or equivalently, how to express the gain due to the use of toes in our walking generation scheme?

\section{A. Compliant toes}

Toe motions cannot be generated independently from the position of the CoP since toe support phases require the $\mathrm{CoP}$ to lie under the toe. In the case of unactuated compliant toe joints, the angle of toe flexion $p_{T}^{\phi}$ is even directly related to the position $z^{x}$ of the CoP relative to the sagittal position $p_{T}^{x}$ of the toe joint $p_{T}$ :

$$
p_{T}^{\phi}:= \begin{cases}K\left(z^{x}-p_{T}^{x}\right) & \text { if } z^{x} \geq p_{T}^{x}, \\ 0 & \text { else }\end{cases}
$$

with $K$ the compliance of the spring in the joint.

But forcing the $\mathrm{CoP}$ to be under the toe in order to generate toe support phases will influence the motion of the CoM, following the dynamics (1), what may perturb the realization of other objectives such as walking with a desired speed or following a position reference of the CoM.

An elegant solution to this problem can be found by looking closer at the feasibility constraint (6) and how it evolves in case of a rotation of angle $p_{T}^{\phi}$ around the support toe:

$$
E_{c}\left(p^{\theta}\right) R_{c}\left(-p_{T}^{\phi}\right)\left(c^{x, y, z}-p_{T}^{x, y, z}\right) \leq e_{c},
$$

where $R_{c}\left(-p_{T}^{\phi}\right)$ rotates the original constraints around the toe joint by the angle $p_{T}^{\phi}$, what is equivalent to a rotation of the CoM by the angle $-p_{T}^{\phi}$. Taking into account this rotation would allow our control scheme to relate toe flexion with the desired motion of the CoM.

In the upper part of Figure 2, we can see the evolution of the frontal part of this constraint for a given CoM position

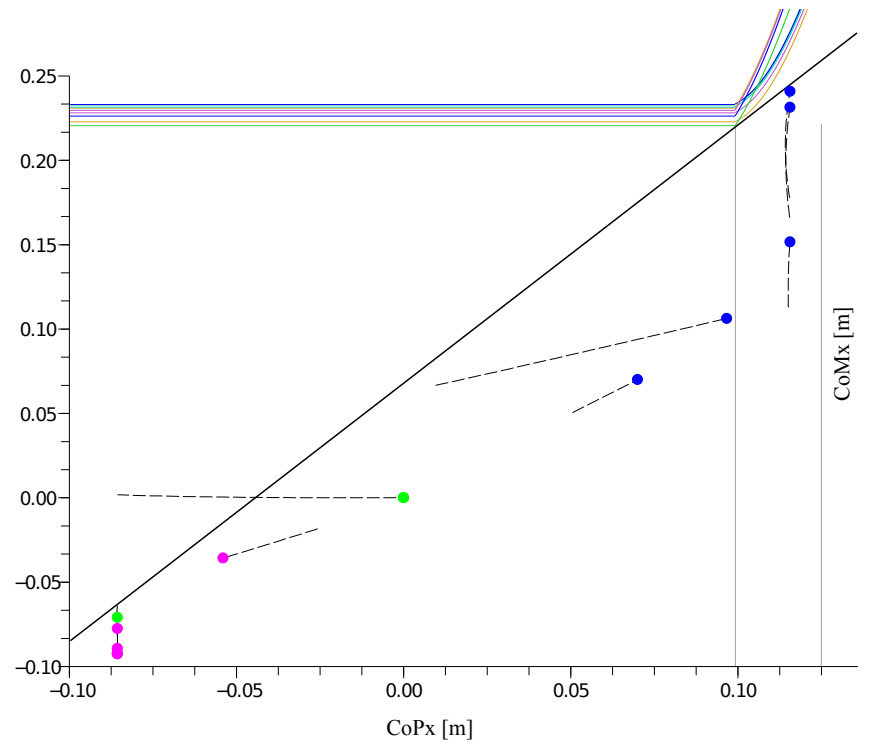

Fig. 2. Co-evolution in the sagittal plane of the CoM (vertically) and the CoP (horizontally) for a maneuver including several walking speeds and directions. The thick dots represent situations at the end of the support phases when the robot is walking in the forward (blue), sideways (green), and backward (magenta) direction, and the dotted lines the situations in the previous $100 \mathrm{~ms}$, when toe flexion may happen. The curves at the top present the nonlinear and nonconvex sagittal evolution of the constraint (14) with a toe flexion following the law (13). Since this constraint needs only be verified at the end of support phases, for situations represented by the thick dots and dotted lines, we can see that a linear approximation of the form (15) indicated by the solid diagonal line does not necessarily overrestrict the set of reachable CoM-positions, making beneficial use of toe flexion when walking forward while impacting backward walking.

and a compliance $K$ such that a maximal toe angle of $45^{\circ}$ is reached when the CoP is at the tip of the toe. We can see that this constraint is nonlinear and non-convex, forbiding its direct inclusion in a linear control scheme. In the lower part of the figure, positions of the CoM relative to the center of the support foot at the end of the support phase, when toe flexion usually occurs, are plotted for several walking speeds and directions. The solid line shows that a linear approximation of the form

$$
\begin{aligned}
& E_{c}\left(p_{T}^{\theta}\right)\left(R_{c}\left(\phi_{*}\right)\left(c^{x, y, z}-p_{T}^{x, y, z}\right)+\right. \\
& \left.\frac{\partial R_{c}\left(\phi_{*}\right)}{\partial \phi}\left(K\left(z^{x}-p_{T}^{x}\right)-\phi_{*}\right)\left(c_{*}^{x, y, z}-p_{T}^{x, y, z}\right)\right) \leq e_{c},
\end{aligned}
$$

could allow a beneficial use of toes while not over-restricting the set of feasible CoM positions when the CoP is away from the toes. Such an approximation reduces the set of feasible positions for the CoM with respect to the original constraint (14), especially when the CoP approaches the heel. The compliance $K$ and the linearization point $\phi_{*}$ must therefore be chosen carefully in order not to hinder specific walking maneuvers.

One useful fact in this regard is that we are mainly interested in toe flexion at the end of the single support phase (see point (b) in the Introduction). The new form (15) of the constraint (6) must therefore replace only the constraint (11) and not the constraint (12), limiting the risk to unnecessarily 
constrain the motion of the CoM because of our linear approximation.

\section{B. Active toes}

On the contrary to passive toes, active toes can control their flexion independently from the position of the CoP, under the condition that it stays under the toe during the entire flexion. A classic inverse kinematic procedure is then sufficient to obtain full-body motion references. We propose however to keep in this case the previous approach for passive compliant toes as a guide to decide whether toe flexion should be realized and to what extent. The active toe only gives in some sense a more controlled way to realize the resulting desired toe motion.

Actuated toe joints introduce furthermore a kinematic redundancy to the legs, which offers the possibility to fulfill supplementary objectives. Similarly to what can be found for example in [19], we choose to minimize the norm of the leg joint velocities $\left\|\dot{q}_{L}\right\|$ :

$$
\operatorname{minimize} \quad\left\|\dot{q}_{L}\right\|^{2}
$$

\section{WALKING EFFICIENCY}

To study the influence of extensions (6) and (15) on the efficiency of the robot motions, we have performed a series of dynamic simulations with varying walking speeds and vertical motions of the CoM (cf. Figure 4). The vertical motion $c^{z}$ in each simulation is the result of adjacent fifth order polynomials connecting predetermined vertical positions, velocities, and accelerations of the CoM at the transition instants $t_{r}$ at the beginning and end of single support phases, varying between the following bounds:

$$
\begin{aligned}
c_{r}^{z} & \in[0.8,0.9] \mathrm{m}, \\
\left\|\dot{c}_{r}^{z}\right\| & \in[0,0.1] \mathrm{m} / \mathrm{s}, \\
\ddot{c}_{r}^{z} & =0 .
\end{aligned}
$$

The desired velocity $\dot{c}_{r e f}^{x}$ of the CoM in the forward direction varies between the following bounds:

$$
\dot{c}_{r e f}^{x} \in[0.1,0.8] \mathrm{m} / \mathrm{s} .
$$

In each simulation, a HRP-2 robot is walking forward for $10 \mathrm{~s}$, trying to realize the specified horizontal velocity for a given vertical evolution of the CoM. Every simulation is performed twice: only with the (static) polyhedral constraints (6), or with the toe variant (15). The resulting motions have been verified against the limitations of the robot, and the realizable motions have been recorded and analyzed.

Two simulated approximations of energy consumptions are considered:

$$
\begin{gathered}
\int_{0}^{10 s}\|\tau(t)\|^{2} d t \\
\int_{0}^{10 s}\left\|\tau(t)^{T} \dot{q}(t)\right\| d t
\end{gathered}
$$

The first criterion reflects the ohmic drop in the joint motors by measuring the torques $\tau$ in the joint, and the second the mechanical work proportional to the joint velocities $\dot{q}$.

Contrary to the second criterion, the first one shows a considerable sensitivity to the variation of the parameters (17) of the vertical motion of the CoM, showing that a significant reduction of the torque load can be achieved by appropriate vertical oscillations. The observed difference between the two criteria is due to the fact that the first one is minimized by an equal distribution of joint torques, whereas the second rises proportionally to angular velocities and torques in every joint. Therefore, the vertical motion alone does not affect the total amount of mechanical work to a considerable extent.

This is different for the use of toes which occurs for higher CoM positions $c_{r}^{z}$, but especially for horizontal CoM velocities above $0.3 \mathrm{~m} / \mathrm{s}$. There again, however, the reduction is significantly stronger for the first criterion, as the use of toe joints lowers the torque peaks in other joints of the stance leg. We can expect to reduce significantly the mechanical work (20) when using passive toes, what however, we have not addressed yet in more details.

On the contrary, both criteria show that the inclusion of toe motions inside our scheme can allow for a decrease in energy consumption for a wider range of displacement velocities if the vertical motions have been chosen properly. The strong fluctuation of the minima in the upper graph of Figure 4 shows, however, that with the chosen range and resolution of boundary conditions (17) this has not been achieved for all realized average velocities. A greater number of simulations is needed to complete these results and to provide a more comprehensive basis for the adaptation scheme mentioned in Section II.

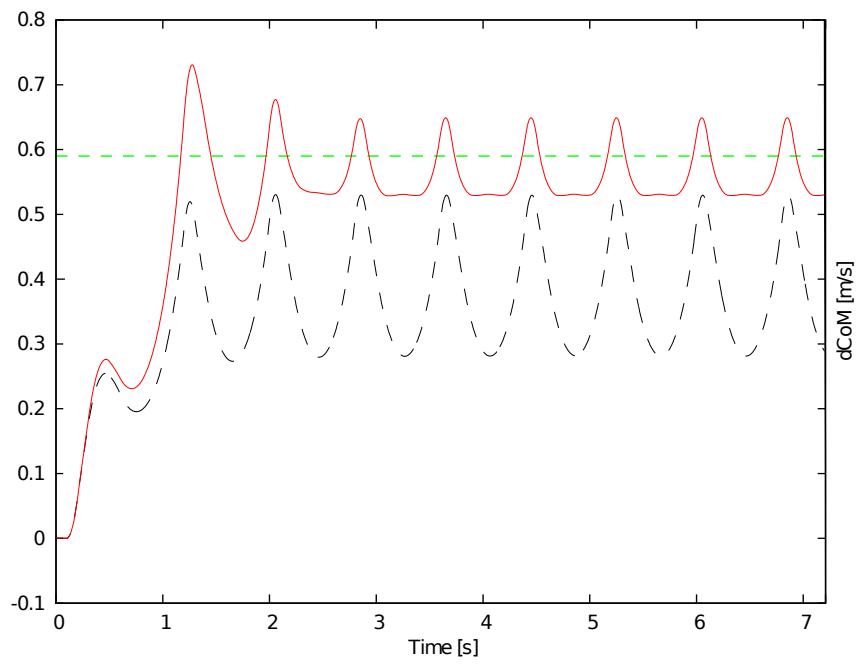

Fig. 3. Attained sagittal CoM velocities with (solid red) and withou (dashed black) the use of toes and polyhedral constraints for a given velocity reference (dashed green).

We can see, finally, that the use of toes allows to realize considerably faster walking speeds $\dot{c}_{r e f}^{x}$ in the forward direction (cf. also Figure 3). If, however, the desired velocity is not realizable for the chosen motion of the CoM, the scheme 

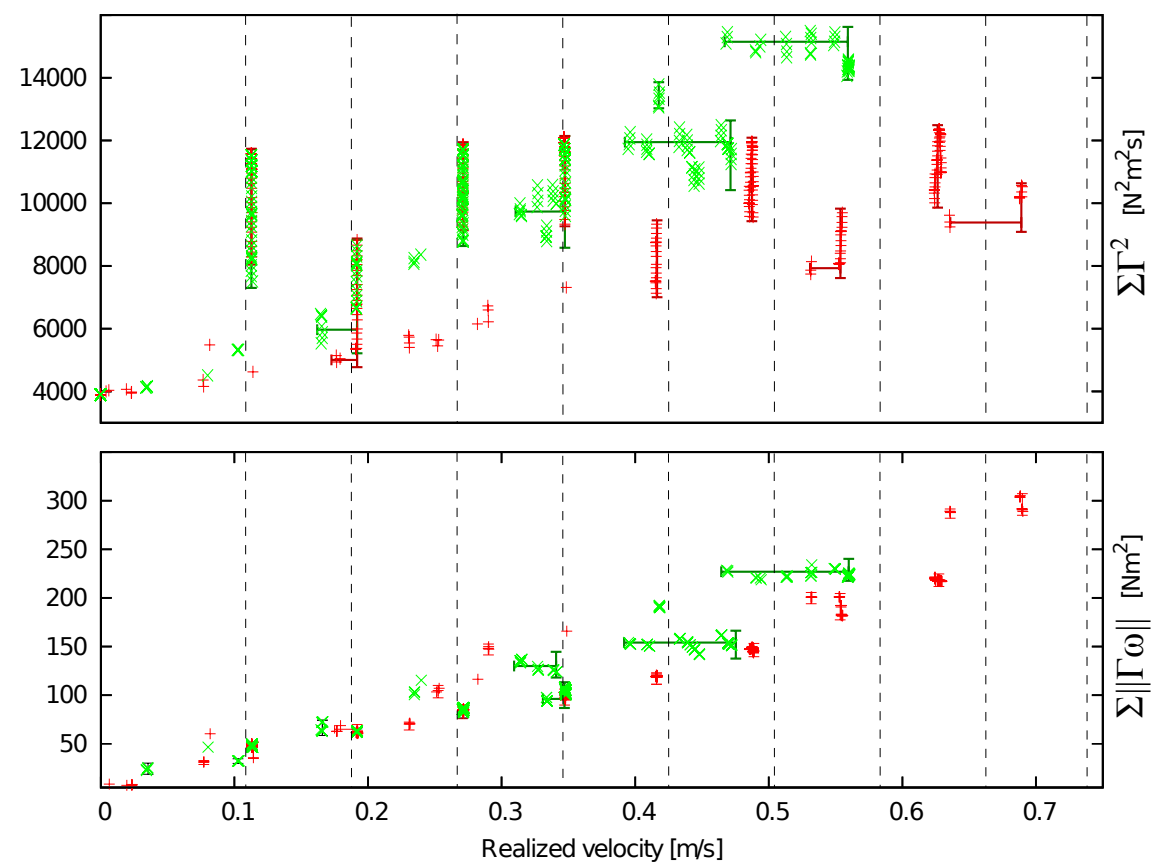

Fig. 4. Energy consumption for realized mean velocities and different vertical motions of the CoM, with the use of toes (red + ) and without (green $\mathrm{x}$ ) as well as different velocity references (dashed black).

manages to reduce the displacement velocity. The polyhedral constraints restrict the set of achievable CoM and foot positions such that only geometrically feasible trajectories are generated. It is, last but not least, for this reason that these constraints constitute an important completion of our trajectory generation scheme. By rendering it more robust to unrealizable references they enforce its most valuable property: The feasibility requirements are always respected, even if it means not to realize a desired motion.

\section{COMPARISON WITH RELATED WORK}

Similar approaches for achieving vertical variations of the CoM can be found in [19][20][21]. There however, the right hand side of Equation (1) is predefined prior to the resolution of the ODEs. The consequence is that no analytical solution can be given for arbitrary vertical variations of the Center of Mass so that numerical integration methods have to be employed or violations of boundary conditions accepted. In our case, CoP, CoM, and foot motions are functions of the control vector, therefore allowing for a seamless implementation of predefined vertical CoM trajectories.

Important consequences of predefining only the vertical motion as a function of time are that, first, only the stepping sequence and not the foot positioning has to be synchronized with the motion of the CoM, and second, the additional computational load is relatively low since the control scheme is kept linear. Approaches predefining the 3D geometry of the motion of the CoM as in [26] lack both of these advantages.

By taking the robot's true geometric limitations into account, the feasibility constraints introduced in this paper allow the robot to operate closer to its limitations, make longer steps, or walk with a higher Center of Mass. The same constraints serve as a criterion for the use of toes. So far, all related approaches lack such a criterion and require predetermined strategies. All control schemes in the literature relate the evolution of the toe angle to predetermined torque trajectories, either directly generating a rotation as soon as the CoP crosses the toe joint [19][24][25], or simply 'accepting' a rotation when passive toes are used [22][23]. In no case, the trajectory of the CoP is adapted online to allow for a desired configuration of the robot.

\section{CONCLUSION}

Reproducing characteristics of the human walk can improve the walking performance of robots. The combination of both the natural vertical motion of the CoM and the use of toes allows to reduce the load on the leg joints and to augment the walking speed. As an essential element of this paper, polyhedral constraints on the position of the CoM allow first to assure the geometric realizability of the CoM and leg motions, and second, to consider the use of toes inside the preview of walking trajectories.

In this paper, vertical motions of the CoM have been given prior to the generation of horizontal trajectories, but the question of how to adapt the vertical variations such that natural and efficient motions can always be obtained has not been answered. More experimental work is necessary to find appropriate adaptation rules. However, we can expect not to find an optimal solution for every situation and the computation of three-dimensional walking trajectories by a unified, nonlinear approach might be necessary to give the biped robot walking the efficiency of the human gait. 


\section{REFERENCES}

[1] S. Kajita, O. Matsumoto, and M. Saigo, "Real-time 3d walking pattern generation for a biped robot with telescopic legs," in IEEE Int. Conf. on Robotics and Automation (ICRA'01), pp. 2299-2306, 2001.

[2] S. Kajita, F. Kanehiro, K. Kaneko, K. Fujiwara, K. Harada, K. Yokoi, and $\mathrm{H}$. Hirukawa, "Biped walking pattern generation by using preview control of zero-moment point," in IEEE Int. Conf. on Robotics and Automation (ICRA'03), vol. 2, pp. 1620-1626, sept. 2003.

[3] M. Morisawa, K. Harada, S. Kajita, K. Kaneko, F. Kanehiro, K. Fujiwara, S. Nakaoka, and H. Hirukawa, "A biped pattern generation allowing immediate modification of foot placement in real-time," in IEEE/RAS Int. Conf. on Humanoid Robots (Humanoids'06), pp. 581586, 2006.

[4] T. Takenaka, T. Matsumoto, and T. Yoshiike, "Real time motion generation and control for biped robot -1st report: Walking gait pattern generation-," in IEEE/RSJ Int. Conf. on Intelligent Robots and Systems (IROS'09), pp. 1084-1091, 2009.

[5] K. Harada, S. Kajita, K. Kaneko, and H. Hirukawa, "An analytical method on real-time gait planning for a humanoid robot," in IEEE/RAS Int. Conf. on Humanoid Robots (Humanoids'04), pp. 640-655, 2004.

[6] K. Nishiwaki, S. Kagami, Y. Kuniyoshi, M. Inaba, and H. Inoue, "Online generation of humanoid walking motion based on a fast generation method of motion pattern that follows desired zmp," in IEEE/RSJ Int. Conf. on Intelligent Robots and Systems (IROS'02), pp. 2684-2689, 2002.

[7] A. Herdt, N. Perrin, and P.-B. Wieber, "Walking without thinking about it," in IEEE Int. Conf. on Intelligent Robots and Systems (IROS'10), pp. 190-195, 2010.

[8] J. M. Donelan, R. Kram, and A. D. Kuo, "Mechanical work for stepto-step transitions is a major determinant of the metabolic cost of human walking," Journal of Experimental Biology, vol. 205, no. 23, pp. 3717-3727, 2002.

[9] K. E. Gordon, D. P. Ferris, and A. D. Kuo, "Metabolic and mechanical energy costs of reducing vertical center of mass movement during gait," Archives of Physical Medicine and Rehabilitation, vol. 90, no. 1, pp. 136-144, 2009.

[10] J. D. Ortega and C. T. Farley, "Minimizing center of mass vertical movement increases metabolic cost in walking," Journal of Applied Physiology, vol. 99, no. 6, pp. 2099-2107, 2005.

[11] P. G. Adamczyk, S. H. Collins, and A. D. Kuo, "The advantages of a rolling foot in human walking," Journal of Experimental Biology, vol. 209, no. 20, pp. 3953-3963, 2006.

[12] A. Kuo, "The six determinants of gait and the inverted pendulum analogy: A dynamic walking perspective," Human Movement Science, vol. 26, no. 4, pp. 617-656, 2007.

[13] M. Franken, G. van Oort, and S. Stramigioli, "Analysis and simulation of fully ankle actuated planar bipedal robots," in IEEE/RSJ Int. Conf. on Intelligent Robots and Systems (IROS'08), pp. 634-639, 2008.

[14] M. Srinivasan and A. Ruina, "Computer optimization of a minimal biped model discovers walking and running," Nature, vol. 439, no. 7072, pp. 72-75, 2006.

[15] D. Tlalolini, C. Chevallereau, and Y. Aoustin, "Comparison of different gaits with rotation of the feet for a planar biped," Robotics and Autonomous Systems, vol. 57, no. 4, pp. 371-383, 2009.

[16] A. D. Kuo, J. M. Donelan, and A. Ruina, "Energetic consequences of walking like an inverted pendulum: Step-to-step transitions," Exercise and Sport Sciences Reviews, vol. 33, no. 2, 2005.

[17] P. G. Adamczyk and A. D. Kuo, "Redirection of center-of-mass velocity during the step-to-step transition of human walking," Journal of Experimental Biology, vol. 212, no. 16, pp. 2668-2678, 2009.

[18] A. D. Kuo, "Energetics of actively powered locomotion using the simplest walking model," Journal of Biomechanical Engineering, vol. 124, no. 1, pp. 113-120, 2002.

[19] Buschmann, Simulation and Control of Biped Walking Robots. PhD thesis, Technische Universität München, 2010.

[20] K. Terada and Y. Kuniyoshi, "Online gait planning with dynamical 3dsymmetrization method," in IEEE/RAS Int. Conf. on Humanoid Robots (Humanoids'07), pp. 222-227, 2007.

[21] R. Tajima, D. Honda, and K. Suga, "Fast running experiments involving a humanoid robot," in IEEE Int. Conf. on Robotics and Automation (ICRA'09), pp. 1571-1576, 2009.

[22] R. Sellaouti, O. Stasse, S. Kajita, K. Yokoi, and A. Kheddar, "Faster and smoother walking of humanoid HRP-2 with passive toe joints," in IEEE/RSJ Int. Conf. on Intelligent Robots and Systems (IROS'06), pp. 4909-4914, 2006.
[23] S. Kajita, S. Kajita, K. Kaneko, M. Morisawa, S. Nakaoka, and H. Hirukawa, "ZMP-based biped running enhanced by toe springs," in IEEE Int. Conf. on Robotics and Automation (ICRA'07), pp. 39633969, 2007.

[24] K. Nishiwaki, S. Kagami, Y. Kuniyoshi, M. Inaba, and H. Inoue, "Toe joints that enhance bipedal and fullbody motion of humanoid robots," in IEEE Int. Conf. on Robotics and Automation (ICRA'O2), pp. 31053110, 2002.

[25] K. Miura, M. Morisawa, F. Kanehiro, S. Kajita, K. Kaneko, and K. Yokoi, "Human-like walking with toe supporting for humanoids," in IEEE/RSJ Int. Conf. on Intelligent Robots and Systems (IROS'11), pp. 4428-4435, 2011.

[26] M. Morisawa, S. Kajita, K. Kaneko, K. Harada, F. Kanehiro, K. Fujiwara, and H. Hirukawa, "Pattern generation of biped walking constrained on parametric surface," in IEEE Int. Conf. on Robotics and Automation (ICRA'05), pp. 2405-2410, 2005. 